\title{
COUNTABLE TIGHTNESS AND PROPER FORCING
}

\author{
Z. BALOGH, A. DOW, D. H. FREMLIN AND P. J. NYIKOS
}

One of the most basic and natural generalizations of first countability is countable tightness: the condition that, whenever $x$ is in the closure of $A$, there is a countable subset $B$ of $A$ such that $x \in \bar{B}$. Countably tight spaces include sequential spaces, i.e., those in which closure is obtainable by iteration of the operation of taking limits of convergent sequences. The two classes are distinct, since there are easy examples of countable, nondiscrete spaces with only trivial convergent sequences. On the other hand, it was long a major unsolved problem whether every compact Hausdorff space of countable tightness is sequential. First posed in [1] and motivated by the main results of [2], it gained importance from subsequent discoveries on the strong structural properties enjoyed by compact sequential spaces (see [3] and its references). A negative answer was shown to be consistent by Ostaszewski [4], who used Gödel's Axiom of Constructibility $(V=L)$ to construct a countably compact space $X$ whose one-point compactification is countably tight; of course, no sequence from $X$ can converge to the extra point. Now (Theorem 2) we have shown that a positive answer follows from the Proper Forcing Axiom (PFA), introduced in [5]. Our research has uncovered many other striking consequences of PFA, numbered below. None was known to be consistent until now, nor was the following consequence of Corollary 1 and Theorem 3: the ordinal space $\omega_{1}$ embeds in every first countable, countably compact, noncompact $T_{2}$ space (in particular, in every countably compact nonmetrizable $T_{2}$ manifold), assuming PFA. This remains true if "first countable" is weakened to "character $\leq \omega_{1}$," meaning every point has a local base of cardinality $\leq \omega_{1}$. This leads to a remarkable structure theorem for regular, countably compact spaces of character $\leq \omega_{1}$ (e.g. the product space $\left.[0,1]^{\omega_{1}}\right)$ : under PFA, the closure of every set $A$ can be taken by first adjoining all limits of convergent sequences, and then adjoining to the resulting set $A^{\wedge}$ all points $x$ for which there is a copy $W$ of $\omega_{1}$ in $A^{\wedge}$ such that $W \cup\{x\}$ is homeomorphic to $\omega_{1}+1$.

Although large cardinals are needed to prove the consistency of PFA, all our PFA results are consistent if $\mathrm{ZF}$ is consistent. This is established by using $\omega_{2}$-p.i.c. [6, Chapter VIII] posets and a ground model with a $\diamond_{\omega_{2}}$-sequence to capture approximations to possible counterexamples in a countable support

Received by the editors August 13, 1987 and, in revised form, February 4, 1988.

1980 Mathematics Subject Classification (1985 Revision). Primary 54D30; Secondary 54A25, 54A35, 03E35, 03E50, 03E65.

Research of the second author supported by NSERC of Canada.

Research of the fourth author partially supported by NSF grant DMS-8503391, and an SERC grant while at the University of Oxford. 
iteration of length $\omega_{2}$, in which all models except the final one (which satisfies $\mathfrak{c}=\omega_{2}$ ) satisfy $\mathrm{CH}$.

As usual, $c$ stands for the cardinality of the continuum. From now on, "space" will mean "regular Hausdorff space".

The concept of a free sequence in a space $X$ is central to our results. This is a sequence $\left\langle x_{\xi}: \xi<\alpha\right\rangle$ such that the closures of $\left\{x_{\eta}: \eta<\xi\right\}$ and $\left\{x_{\gamma}: \gamma \geq \xi\right\}$ are disjoint for each $\xi<\alpha$. We use

THEOREM A [3]. The tightness of a compact space is the supremum of the cardinalities of its free sequences.

A space is called $\alpha$-realcompact if every maximal family of closed sets with the countable intersection property has nonempty intersection.

THEOREM 1 [PFA]. Every space is either $\alpha$-realcompact or has an uncountable free sequence.

A space is compact if, and only if, it is $\alpha$-realcompact and countably compact. A simple inductive construction gives:

COROLLARY 1 [PFA]. A countably compact space $X$ is either compact or contains a closed preimage of $\omega_{1}$ (i.e., a subspace which admits a closed continuous map onto $\left.\omega_{1}\right)$. If $X$ is countably tight, then the subspace can be made closed in $X$.

A continuous preimage of $\omega_{1}$ has an open cover of cardinality $\omega_{1}$ with no finite subcover; in other words, the preimage fails to be initially $\omega_{1}$-compact.

COROLlARY 2 [PFA]. If $X$ is countably tight and either (i) initially $\omega_{1}$ compact or (ii) normal, separable, and countably compact, then $X$ is compact.

THEOREM B [7]. If $M A$, then a compact space is sequential iff every countably compact subspace is compact.

A second application of PFA gives:

THEOREM 2 [PFA]. Every compact space of countable tightness is sequential.

OUTLINE OF PROOF. Given a compact nonsequential space $Z$ and a closed surjective $f: Y \rightarrow \omega_{1}, Y \subset Z$, given by Corollary 1 and Theorem B, assign open neighborhoods $W(y), V(y)$ in $Z$ for each $y \in Y$, with $\operatorname{cl}_{Z} V(y) \subset W(y)$ and $\mathrm{cl}_{Z} W(y)$ disjoint from $\left\{f^{-1}(\gamma): \gamma>f(y)\right\}$. A proper poset then produces, assuming PFA, a function $h: \omega_{1} \rightarrow Y$ such that $h(\xi) \in V(h(\eta))$ whenever $\eta<\xi<\omega_{1}$, but with $h^{-1}[V(h(\xi))]$ open in $\omega_{1}$ for every $\xi<\omega_{1}$. This gives the image of $[0, \xi]$ a finite cover by sets of the form $V(h(\xi))$, so that its closure in $Z$ is a subset of $\bigcup\{W(h(\eta)): \eta \leq \xi\}$. This makes the image of $\omega_{1}$ a free sequence in $Z$, contradicting its countable tightness.

A natural choice for the above poset is the set of all finite approximations to $h$, ordered by $g \leq f$ iff $g$ extends $f$ and, if $\eta<\xi, \eta \in \operatorname{dom} g, \xi \in \operatorname{dom} f$, then either $g(\eta) \in V(f(\xi))$ or there is already $\gamma \in \operatorname{dom} f \cap[\eta, \xi)$ such that $f(\gamma) \notin V(f(\xi))$. For dense sets the natural choice would be, given $\alpha<\omega_{1}$, 
$D_{\alpha}=\{f: \gamma \in \operatorname{dom} f$ for some $\gamma \geq \alpha\}$. A filter generic for the $D_{\alpha}$ (see [5] for definitions) straightforwardly gives $h$.

In many cases, unfortunately, the above poset is not proper, so we add side conditions in the manner of [8]. This complicates the definition, and the proof of properness, incorporating ideas from [8] and $\S \S 3$ and 5 of [5], is very technical.

In proving Theorem 1 , the role of $f$ is, roughly speaking, taken over by a maximal family $\mathbf{F}$ of closed sets with c.i.p. and empty intersection. PFA is used for the case where $\mathbf{F}$ has a base (in the filter sense) of separable sets. A straightforward ZFC induction takes care of the otherwise case.

If the space $Y$ above has character $\leq \omega_{1}$, we can build a local base at each point into the poset and the generic $h$ will embed $\omega_{1}$ into $Y$, giving:

THEOREM 3 [PFA]. Every closed preimage of $\omega_{1}$ of character $\leq \omega_{1}$ contains a copy of $\omega_{1}$.

Corollaries of Theorems 2 and 3 abound. For instance, we can replace "perfect preimage of $\omega_{1}$ " by "copy of $\omega_{1}$ " wherever it appears as a hypothesis in [9], assuming PFA. A striking example is

COROLlARY 3 [PFA]. Let $X$ be a locally compact, first countable space of cardinality $\leq \omega_{1}$. Exactly one of the following is true.

(a) $X$ contains a copy of $\omega_{1}$.

(b) $X$ is a countable union of closed discrete subspaces (hence a Moore space).

Since one-point compactifications neither create nor destroy perfect preimages of $\omega_{1}$, we have

COROLLARY 4 [PFA]. If a locally compact space (i) has a countably compact noncompact subspace or (ii) is separable and has cardinality $>\mathfrak{c}$, then it contains a perfect preimage of $\omega_{1}$.

Part (ii) uses a well-known fact about sequential spaces [3]: every countable subset has closure of cardinality $\leq \mathbf{c}$.

Our numbered results are independent of ZFC. With the exception of Corollary 2(i) [one of us has a model of MA where it fails to hold], this can be demonstrated by a number of examples of the 1970s using $\diamond$, a consequence of $V=L$ : (a) Ostaszewski's space [4] which is locally compact, noncompact, hereditarily separable (hence admits no continuous map onto $\omega_{1}$, yet has countably tight compactification), countably compact, perfectly normal, and of cardinality $\omega_{1}$; (b) A principal $S^{1}$-bundle over the long ray (6.17 of [10]) with a perfect preimage (but no copy) of $\omega_{1}$ and (c) Fedorchuk's [11] compact hereditarily separable space of cardinality $2^{\mathfrak{c}}$ with no nontrivial convergent sequences.

\section{REFERENCES}

1. R. C. Moore and S. G. Mrówka, Topologies determined by countable objects, Notices Amer. Math. Soc. 11 (1964), 554.

2. H. S. Bear and B. Yood, Multiplicative functionals on semigroups of continuous functions, Proc. Amer. Math. Soc. 10 (1959), 736-741. 
3. A. V. Arhangel'skii, Structure and classification of topological spaces and cardinal invariants, Uspekhi Mat. Nauk. 33: 6 (1978), 29-84 = Russian Math. Surveys 33: 6 (1978), 33-96.

4. A. Ostaszewski, On countably compact, perfectly normal spaces, J. London Math. Soc. (2) 14 (1976), 505-516.

5. J. E. Baumgartner, Applications of the proper forcing axiom, Handbook of Set-Theoretic Topology (K. Kunen and J. Vaughan, eds.), North-Holland, 1984, pp. 913-959.

6. S. Shelah, Proper forcing, Lecture Notes in Math., vol. 940, Springer-Verlag, Berlin and New York, 1982.

7. P. Nyikos and M. Ismail, On spaces in which countably compact subsets are closed, and hereditary properties, Topology Appl. 11 (1980), 281-292.

8. S. Todorcevic, A note on the proper forcing axiom, Contemp. Math., vol. 31, Amer. Math. Soc., Providence, R. I., 1984, pp. 209-218.

9. Z. Balogh, Locally nice spaces under Martin's Axiom, Comment. Math. Univ. Carolinae 24 (1983), 63-87.

10. P. Nyikos, The theory of nonmetrizable manifolds, Handbook of Set-Theoretic Topology (K. Kunen and J. Vaughan, eds.), North-Holland, 1984, pp. 633-684.

11. V. V. Fedorchuk, Fully closed mappings and a compatibility of some theorems of general topology with the axioms of set theory, Math. USSR-Sb. 99 (1976), 3-33.

Department of Mathematics, Kossuth University, H-4010 Debrecen, HUNGARY

Department of MAThematics, York University, Downsview, Ontario, CANADA M3J 1P3

DePARTMENT OF MATHEMATICS, UNIVERSity OF ESSEX, COLCHESTER C04 3SQ, ENGLAND

Department of Mathematics, UniVersity of South Carolina, Columbia, SOUTH CAROLINA 29208 